09-04

\section{Plasma apolipoprotein E: roles and targets in schizophrenia and bipolar disorder}

\author{
S Sundram ${ }^{1,2,3}$ \\ 'Northern Psychiatry Research Centre; 'Molecular Psychopharmacology, \\ Mental Health Research Institute of Victoria; and ${ }^{3}$ Department of Psychiatry, \\ The University of Melbourne, Melbourne, Victoria, Australia
}

Apolipoprotein E (apoE) belongs to a large heterogeneous family of lipid-binding proteins and is produced by a variety of tissues including the brain and liver where it subserves a diversity of functions. The primary metabolic role of apoE is as a key regulator of cholesterol and triglyceride transport between cells. It has three isoforms, E2, E3 and E4, and genetic variation in the distribution of these isoforms are associated with plasma lipid levels and atherosclerosis; the $\mathrm{E} 4$ isoform is also associated with increased risk for Alzheimer's disease, impaired cognition and decreased neurite outgrowth. As both lipid metabolism and synaptic connectivity are disturbed in schizophrenia and bipolar disorder, the role of apoE has been investigated in these disorders. The majority of studies have focused on the association between the genetic polymorphisms of apoE and schizophrenia with inconsistent results; however, measurement of protein apoE levels in the brain report changes in both disorders. We report here the first study to our knowledge of measurement of plasma apoE levels in subjects with schizophrenia and bipolar disorder in a medication-free state and following 6 weeks of treatment. In both medication-free schizophrenia and bipolar disorder subjects, plasma apoE levels were significantly decreased compared with control subjects and although there were increases in both groups following treatment, levels were still significantly less than for the control group. These results will be discussed in reference to their implications for altered lipid metabolism in these two disorders and the potential for the development of therapeutics to rectify these deficits leading to improved health outcomes for people with schizophrenia and bipolar disorder.

\section{Identifying Gene-brain Markers of Cognition and Emotion: Implications for Psychiatric Disorders}

\section{Williams}

The Brain Dynamics Centre, Westmead Millennium Institute, Westmead Hospital and Discipline of Psychological Medicine, University of Sydney, Australia

\section{Overview}

This symposium reviews the biological underpinnings of particular genes and will examine biological markers of emotional and cognitive dysfunction in healthy samples. The data presented will be drawing from a standardized integrative database that includes measures of neural and autonomic activity, and behaviour. In particular, the interacting effects of environmental influences (stress) will be examined, as well as moderating effects of gender. This work was supported by an ARC-linkage grant (LP0455104).

$10-01$

\section{Understanding genotype-phenotype relationships using the Brain Resource International Database: Implications for psychiatric conditions}

\author{
C Dobson-Stone', JM Gatt², S Kuan², RH Paul'3, \\ E Gordon $^{4}$, LM Williams ${ }^{2}$, PR Schofield ${ }^{5}$
}

'Prince of Wales Medical Research and Garvan Institutes, Australia; ${ }^{2}$ The Brain Dynamics Centre, Westmead Millennium Institute, Westmead Hospital and Western Clinical School, University of Sydney, Australia; ${ }^{3 B}$ rown Medical School, USA; ${ }^{4}$ The Brain Resource International Database (The Brain Resource Company), Australia; and ${ }^{5}$ Prince of Wales Medical Research and Garvan Institutes, Australia

Background: Psychiatric disorders are characterized by breakdowns in higher brain functions (eg, memory, attention, emotion), which have a substantial genetic component. Elucidating the relationship between genetic variation and brain function variation is an important step in understanding the dispositions to psychiatric disorder. Some functional polymorphisms that have consequences for aspects of brain function will be described. These include the Val66Met substitution in brain-derived neurotrophic factor, the Val108/ $158 \mathrm{Met}$ substitution in catechol-o-methyl transferase, the $\varepsilon 2 / 3 / 4$ polymorphism in apolipoprotein $E$, the serotonin transporter promoter deletion polymorphism and a variable number tandem repeat (VNTR) polymorphism that alters expression of monoamine oxidase $\mathrm{A}$ (MAOA).

Methods: We are investigating these variants using the Brain Resource International Database (BRID), a standardized and integrated database of brain function from healthy volunteers and targeted clinical groups. Data collected include neuropsychological and DASSNEO test scores, cognition and emotion event-related potentials, autonomic measures, and structural and functional magnetic resonance imaging.

Results: This approach is illustrated by our investigation of the MAOA VNTR polymorphism in 312 BRID 